\title{
The use of spine stereotactic radiosurgery for oligometastatic disease
}

\author{
Jennifer C. Ho, MD, ${ }^{1}$ Chad Tang, MD, ${ }^{1}$ Brian J. Deegan, MD, PhD, ${ }^{1}$ Pamela K. Allen, PhD, ${ }^{1}$ \\ Eric Jonasch, MD, ${ }^{2}$ Behrang Amini, MD, PhD, ${ }^{3}$ Xin A. Wang, PhD, ${ }^{4}$ Jing Li, MD, PhD, ${ }^{1}$ \\ Claudio E. Tatsui, MD, ${ }^{5}$ Laurence D. Rhines, MD, ${ }^{5}$ Paul D. Brown, MD, ${ }^{1}$ and Amol J. Ghia, MD ${ }^{1}$ \\ Departments of ${ }^{1}$ Radiation Oncology, ${ }^{2}$ Genitourinary Medical Oncology, ${ }^{3}$ Diagnostic Radiology, ${ }^{4}$ Radiation Physics, and \\ ${ }^{5}$ Neurosurgery, The University of Texas MD Anderson Cancer Center, Houston, Texas
}

\begin{abstract}
OBJECTIVE The authors investigated the outcomes following spine stereotactic radiosurgery (SSRS) for patients with oligometastatic disease of the spine.

METHODS The study was a secondary analysis of 38 of 209 patients enrolled in 2 separate institutional Phase I/II prospective protocols and treated with SSRS between 2002 and 2011. Of these 38 patients, 33 (87\%) were treated for a solitary spine metastasis, with no other history of metastatic disease. SSRS was prescribed to 24 Gy in 1 fraction (8\%), $18 \mathrm{~Gy}$ in 1 fraction (18\%), 16 Gy in 1 fraction (11\%), 27 Gy in 3 fractions (53\%), 30 Gy in 5 fractions (8\%), or 20 Gy in 5 fractions (3\%). Seventeen patients (45\%) received prior conventional external beam radiation therapy.

RESULTS The median overall survival (OS) was 75.7 months, and the 2- and 5-year OS rates were $84 \%$ and $60 \%$, respectively. In multivariate analysis, patients who had prior spine surgery and a better Karnofsky Performance Scale score had an improved OS (HR 0.16, 95\% Cl 0.05-0.52, $\mathrm{p}<0.01$, and HR 0.33, 95\% Cl 0.13\%-0.84\%, $\mathrm{p}=0.02$, respectively), and those who had undergone prior radiation therapy had a worse OS (HR 3.6, 95\% Cl 1.2\%-10\%, $p=0.02$ ). The 1-, 2-, and 5-year local progression-free survival rates were $85 \%, 82 \%$, and $78 \%$, respectively. The median time to systemic therapy modification was 41 months. Two patients (5\%) experienced late Grade 3-4 toxicity.

CONCLUSIONS Patients with oligometastatic disease of the spine treated with SSRS can experience long-term survival and a long time before needing a modification in systemic therapy. In addition, SSRS leads to excellent local control and minimal late toxicity.
\end{abstract}

http://thejns.org/doi/abs/10.3171/2016.1.SPINE151166

KEY WORDS spine metastases; oligometastic disease; radiosurgery; oncology

$\mathrm{S}$ PINE metastases are the most common site of osseous cancer metastases, with more than 180,000 new cases per year occurring in North America, and in approximately $40 \%$ of patients with metastatic cancer with spinal metastases. ${ }^{12,22,32}$ External beam radiation therapy (EBRT) with conventional fractionation has historically played a large role in the treatment of spine metastases, with a complete response rate for pain of about $13 \%-$ $18 \% .^{9,15}$ Because patients with metastatic cancer are living longer, the incidence of spine metastases is expected to increase, creating the need for effective, durable treatment results.
Spine stereotactic radiosurgery (SSRS) is becoming accepted as a method of delivering an ablative dose of radiation therapy in a single or limited number of fractions, with a high degree of conformity allowing for treatment near the spinal cord and other critical structures. It appears to lead to improved tumor control and pain control rates compared with conventionally dosed palliative EBRT, especially for radioresistant histologies, although there has not been any randomized evidence to date..$^{13,23,31}$ In addition, it has proven to be an effective salvage tool in patients who have progressive spinal metastases who have received prior radiation. ${ }^{11,13,31}$

ABBREVIATIONS $\mathrm{Cl}=$ confidence interval; $\mathrm{CTV}=$ clinical treatment volume; $\mathrm{DFS}=$ disease-free survival; Dmax = dose maximum; Dmin = dose minimum; EBRT = external beam radiation therapy; GI = gastrointestinal; GTV = gross tumor volume; HR = hazard ratio; KPS = Karnofsky Performance Scale; OS = overall survival; SSRS = spine stereotactic radiosurgery; STMFS = systemic therapy modification-free survival.

SUBMITTED September 30, 2015. ACCEPTED January 15, 2016.

INCLUDE WHEN CITING Published online April 1, 2016; DOI: 10.3171/2016.1.SPINE151166. 
In general, patients with oligometastatic disease have been shown to have better prognoses than those with multiple metastatic sites, with some able to attain very long-term survival. ${ }^{26,27}$ Patients specifically with spine oligometastatic disease may have a more favorable survival compared with patients with synchronous metastatic disease in other sites, and are thus likely to benefit from an ablative rather than a palliative dose of radiation. Moreover, definitive treatment of oligometastatic disease may delay the initiation of or change in systemic therapy. We therefore sought to investigate the long-term outcomes of patients treated with SSRS for oligometastatic disease, including their survival, recurrence patterns, time to systemic therapy modification, and long-term toxicity.

\section{Methods}

\section{Patient Population}

After institutional review board approval, we reviewed the records of 209 patients who were enrolled in two Phase I/II trials between 2002 and 2011, evaluating single and multifraction SSRS for the treatment of spinal metastases at our institution. ${ }^{4,10,11}$ Protocol inclusion criteria were a Karnofsky Performance Scale (KPS) score $>40$, histopathological confirmation of cancer at our institution, and MRI identifying spinal or paraspinal metastasis $<1$ month prior to enrollment. Protocol exclusion criteria were spinal cord compression and unstable spine as determined by multidisciplinary evaluation, cytotoxic chemotherapy within 1 month of enrollment, or EBRT in the prior 3 months to the same site. Before treatment, all patients were presented at a multidisciplinary tumor board to assess appropriate candidacy for the use of SSRS.

We selected 38 patients who had been treated for oligometastatic disease of the spine for this secondary analysis. Patients were included if the spinal metastasis treated with SSRS was the only known site of active progressive disease at the time of treatment. Five patients were included who had a history of previous metastases that had been treated earlier and that were no longer active at the time of SSRS.

\section{Treatment}

All patients underwent CT-guided intensity modulated stereotactic body radiation therapy with the CT-on-rails EXaCT targeting system or Trilogy treatment delivery system with On-board Cone Beam CT (Varian Medical Systems), as previously described. ${ }^{4}$ Patients were immobilized using an Elekta BodyFix stereotactic body frame system (Elekta). Treatment planning was performed using intensity-modulated radiation therapy inverse-treatment planning software (Pinnacle, Philips Medical Systems). Each treatment was monitored by the treating radiation oncologist and a dedicated radiation physicist to verify target positioning and quality assurance, respectively.

SSRS was prescribed to the gross tumor volume (GTV), which was delineated based on the MRI fused with the planning CT. The clinical treatment volume (CTV) included the GTV along with contiguous bone marrow that was at risk. The GTVs were prescribed to receive $20 \mathrm{~Gy}$ in 5 fractions or $30 \mathrm{~Gy}$ in 5 fractions prior to transitioning to
27 Gy in 3 fractions on the multifraction protocol, which included patients who had received up to 1 previous course of spine radiotherapy to the same region (from prior spine radiation or radiation to another site that had exposed their spinal cord at that level). ${ }^{1}$ SSRS was not given as a boost in any case. Patients on the subsequent single-fraction protocol, which excluded patients who had undergone spine radiation in the same region, received 16 Gy to 24 Gy, depending on histology. The biologically equivalent maximum dose (Dmax) for cord constraint on the multifraction protocol was $10 \mathrm{~Gy}$ for the 5 -fraction treatment and $9 \mathrm{~Gy}$ for the 3-fraction treatment. Cord constraint on the single-fraction protocol was $0.01 \mathrm{~cm}^{3}<10 \mathrm{~Gy}$. Multifraction treatments were administered on alternating days.

\section{Follow-Up and End Points}

Per protocol, patients underwent MRI of the spine with and without contrast and were evaluated at clinic visits 3 , $6,9,12,18$, and 24 months following treatment, and every 6 months thereafter. Patients also had other surveillance imaging performed, such as CT, PET/CT, and bone scans, ordered at the discretion of their medical oncologist. Patients unable to return for follow-up were contacted annually to obtain information about survival, disease, and treatment status.

End points assessed included overall survival (OS), local control, disease-free survival (DFS), systemic therapy modification-free survival (STMFS), and toxicity. Local recurrences were identified on follow-up spine MRI by the reading radiologist and confirmed by a radiation oncologist after review of the original SSRS treatment plan. The date of systemic therapy modification was the date that patients were initiated on or had a modification in their systemic treatment regimen after SSRS by the treating medical oncologist, usually for progressive disease. Systemic therapy included cytotoxic chemotherapy, targeted agents, hormonal therapy, and radioactive iodine. For patients who underwent systemic therapy that was planned to begin after SSRS, the date of the next modification of systemic treatment was used. Systemic therapy modification due to patient intolerance was not scored. Toxicity was scored using the Common Terminology Criteria for Adverse Events version 4.0. Toxicity was recorded if radiation therapy was a possible cause, but complications believed to be due to progressive tumors were excluded.

\section{Statistical Analysis}

All survival rates were calculated from the date of SSRS. For local control and progression-free survival patients were censored at the date of last imaging with either MRI or CT. For OS, patients were censored at the date they were last known to be alive. For DFS and STMFS, patients were censored at the date of last follow-up. For DFS, local failure, out-of-field spine failure, distant nonspine metastasis, and death were scored as events. Local failure status was determined based on the patient's spine MRI, and distant nonspine metastasis was based on various other imaging modalities, most commonly $\mathrm{CT}$ or $\mathrm{PET} / \mathrm{CT}$.

Data analysis was performed using Stata/MP statistical 
software (version 14.0, StatCorp). The Fisher's exact test was used to assess measures of association in frequency tables. The equality of group medians was assessed with a nonparametric test for equality. The survival function was performed using Kaplan-Meier estimates. The logrank test was used to assess the equality of the survivor function across groups. A p value $\leq 0.05$ was considered statistically significant. Statistical tests were based on a 2-sided significance level.

The Cox proportional hazard model was used to assess the effect of factors of significance on the survival end points for univariate and multivariate analysis. The estimated hazard ratio (HR) is reported. Multivariate analysis was performed on all factors found to have a $p$ value $\leq 0.25$ on univariate analysis. Backward elimination was performed with the least significant factor eliminated in a stepwise manner until the most significant variables were identified.

\section{Results}

\section{Patient, Tumor, and Treatment Characteristics}

Baseline patient characteristics and treatment characteristics are listed in Table 1. The most common primary histology was renal cell carcinoma (26\%), and $42 \%$ of patients had radioresistant histology (defined as renal cell, sarcoma, and melanoma). The most common dose and fractionation was 27 Gy in 3 fractions (53\%). Seventeen (45\%) and $16(42 \%)$ patients had prior radiation therapy and surgery to the same site, respectively. Eleven patients received a prior course of radiation therapy for spinal metastatic disease at the same level and underwent SSRS for progressive disease. Six patients received a previous course of nonspine radiation (such as for the primary cancer) that had exposed their spinal cord at the same level as that in patients receiving subsequent SSRS. Thirty-three patients $(87 \%)$ had solitary spine metastases, with no prior history of any other metastasis besides the site undergoing SSRS. The other 5 patients (13\%) received SSRS for a single spine metastasis as the only site of active/progressive disease, and had a history of a prior metastasis treated with radiation or surgery.

Three patients $(8 \%)$ were receiving systemic therapy within the 2 months prior to the date of SSRS. Twenty-five patients $(66 \%)$ were not receiving any systemic therapy within this time period. Ten patients $(26 \%)$ were started on a systemic therapy shortly after the completion of SSRS, which was planned prior to SSRS (endocrine therapy, $\mathrm{n}=$ 5; targeted agent, $\mathrm{n}=2$; chemotherapy, $\mathrm{n}=2$, radioactive iodine, $\mathrm{n}=1$ ).

\section{Overall Survival}

At a median follow-up of 69 months (range 9-145 months), 21 patients (55\%) had died. Among patients still alive at the time of this analysis $(\mathrm{n}=17)$, the median follow-up was 75 months (range 53-145 months). The median OS was 75.7 months (Fig. 1). The 1-, 2-, and 5-year survival rates were 95\% (95\% CI 81\%-99\%), 84\% (95\% CI 68\%-93\%), and 60\% (95\% CI 43\%-74\%), respectively. Patients with a KPS score of 70-80 (compared with 90-100) had a worse OS (HR 3.0, 95\% CI 1.2\%-7.5\%,
TABLE 1. Patient and treatment characteristics

\begin{tabular}{|c|c|}
\hline Variable & Value (\%) \\
\hline Median age in yrs (range) & $60(22-88)$ \\
\hline \multicolumn{2}{|l|}{ Race (\%) } \\
\hline White & $31(82)$ \\
\hline Hispanic & $4(11)$ \\
\hline Black & $2(5)$ \\
\hline Asian & $1(3)$ \\
\hline \multicolumn{2}{|l|}{ Radioresistance (\%) } \\
\hline Radioresistant* & $16(42)$ \\
\hline Radiosensitive & $22(58)$ \\
\hline \multicolumn{2}{|l|}{ Primary site (\%) } \\
\hline Renal & $10(26)$ \\
\hline Breast & $7(18)$ \\
\hline Sarcoma & $4(11)$ \\
\hline Lung & $3(8)$ \\
\hline Thyroid & $3(8)$ \\
\hline Other & $11(29)$ \\
\hline \multicolumn{2}{|l|}{ KPS score (\%) } \\
\hline $90-100$ & $20(53)$ \\
\hline $70-80$ & $18(47)$ \\
\hline Prior spine surgery $(\%) \dagger$ & $16(42)$ \\
\hline Prior spine radiation $(\%) \dagger$ & $17(45)$ \\
\hline Systemic therapy prior to SSRS (\%)‡ & $3(8)$ \\
\hline Chemotherapy & $1(3)$ \\
\hline Targeted therapy & $2(5)$ \\
\hline \multicolumn{2}{|l|}{ Type of metastasis (\%) } \\
\hline Solitary§ & $33(87)$ \\
\hline Singleף & $5(13)$ \\
\hline \multicolumn{2}{|l|}{ Spine location (\%) } \\
\hline Cervical & $4(11)$ \\
\hline Lumbar & $13(34)$ \\
\hline Sacral & $1(3)$ \\
\hline Thoracic & $20(53)$ \\
\hline Median tumor vol in $\mathrm{cm}^{3}$ (range) & $28.4(1.6-98.3)$ \\
\hline \multicolumn{2}{|l|}{ Fractionation schemes (\%) } \\
\hline 24 Gy in 1 fraction & $3(8)$ \\
\hline 18 Gy in 1 fraction & $7(18)$ \\
\hline 16 Gy in 1 fraction & $4(11)$ \\
\hline 27 Gy in 3 fractions & $20(53)$ \\
\hline 30 Gy in 5 fractions & $3(8)$ \\
\hline 20 Gy in 5 fractions & $1(3)$ \\
\hline $\begin{array}{l}\text { Median time from cancer diagnosis to SSRS in } \\
\text { mos (range) }\end{array}$ & $40.8(0.9-201)$ \\
\hline \multicolumn{2}{|c|}{$\begin{array}{l}\text { * Defined as renal cell carcinoma, melanoma, and sarcoma. } \\
\dagger \text { Prior treatment at the same site that underwent SSRS. } \\
\text { ‡ Given within } 2 \text { months of SSRS. } \\
\S \text { Only known site of metastatic disease. } \\
\text { ๆ Only active metastasis with history of previously treated metastasis. }\end{array}$} \\
\hline
\end{tabular}




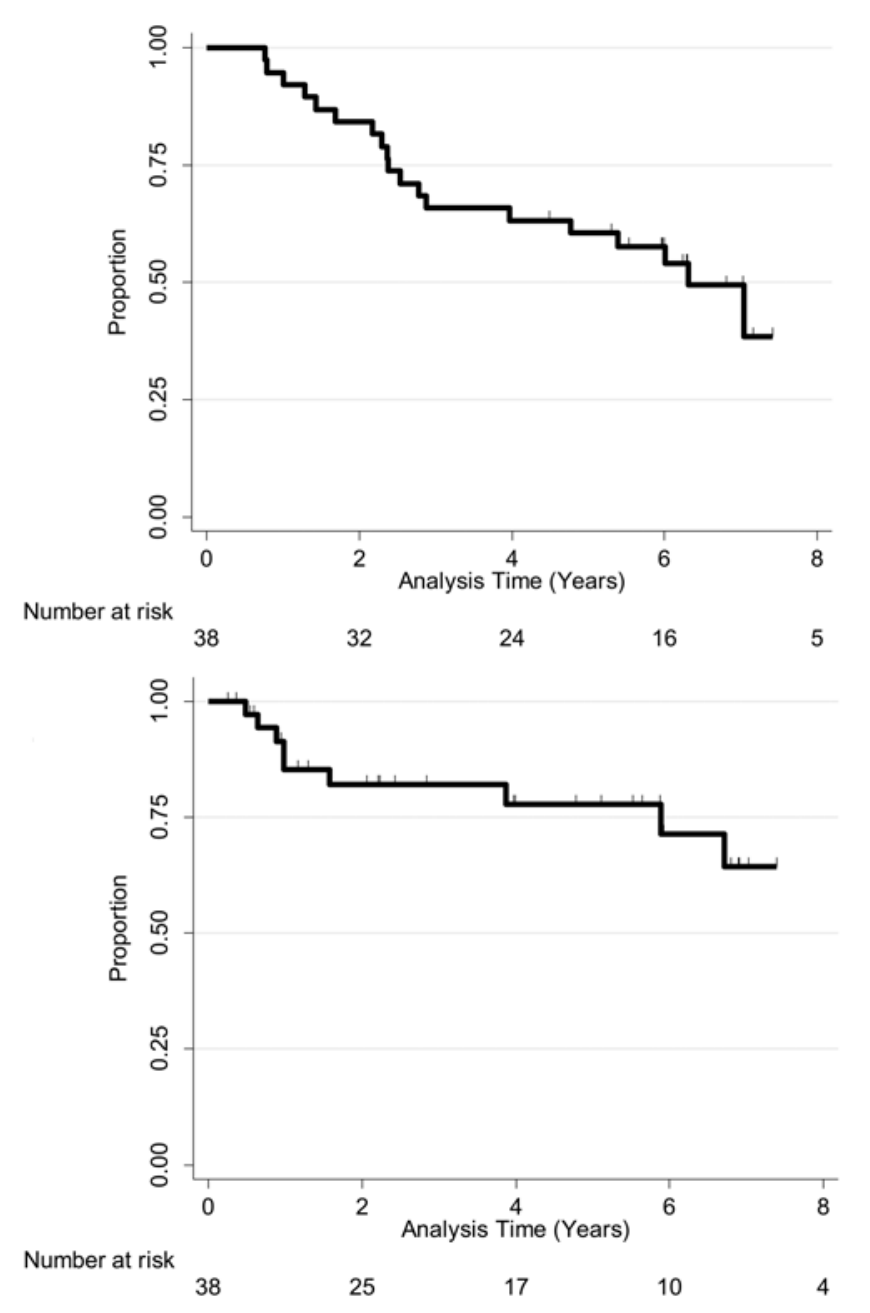

FIG. 1. Kaplan-Meier curves demonstrating OS (upper) and local progression-free survival (lower) in all patients.

$\mathrm{p}=0.02$; Table 2, Fig. 2). Patients who had prior spine surgery had a better OS (HR $0.33,95 \%$ CI $0.12 \%-0.92 \%$, $\mathrm{p}=0.03$; Table 2, Fig. 2). Other factors assessed were not significant, including age, sex, tumor volume, radioresistance, spine location, fractionation, and prior radiation. On multivariate analysis, prior radiation (HR 3.6, 95\% CI $1.2 \%-10 \%, \mathrm{p}=0.02)$, prior surgery (HR $0.16,95 \% \mathrm{CI}$ $0.05 \%-0.52 \%, \mathrm{p}<0.01$ ), and higher KPS score (HR 0.33 , $95 \%$ CI $0.13 \%-0.84 \%, \mathrm{p}=0.02$ ) was a significant predictor (Table 3).

\section{Spinal Recurrence}

The median local progression-free survival was 130.7 months, and the 1-, 2-, and 5-year rates were 85\% (95\% CI $68 \%-94 \%$ ), $82 \%$ (95\% CI 64\%-91\%), and $78 \%$ (95\% CI 59\%-89\%), respectively (Fig. 1). No variables were identified as predictive of local failure in univariate (Table 2) or multivariate analysis (Table 3).

The patterns of spinal recurrence and the subsequent management strategies are summarized in Table 4. Among the 10 patients (26\%) with local progression, 4 underwent surgery, 2 had further surgery and EBRT, 3 had systemic therapy alone, and 1 patient had a repeat course of SSRS ( 27 Gy in 3 fractions, given 1 year after the initial SSRS). Thirteen patients (34\%) experienced out-of-field spine metastases following SSRS at a median of 27.6 months (range 8.5-91.3 months) after SSRS. Three of these 13 patients' subsequent out-of-field spine metastases were located in spinal levels directly adjacent to the site of SSRS and were treated with SSRS in 1 patient, EBRT in 1 patient, and surgery in 1 patient. In the 10 other patients, their distant spine failures were managed with SSRS $(n=5)$, EBRT ( $=1)$, surgery followed by SSRS $(n=1)$, and systemic therapy or best supportive care $(n=3)$.

\section{Disease-Free Survival}

The median DFS was 19.0 months. The 1- and 2-year DFS rates were $62 \%$ (95\% CI 44\%-75\%) and 44\% (95\% CI $28 \%-60 \%$ ), respectively. Patients with a lower KPS score (70-80 vs 90-100) had a worse DFS (HR 2.3, 95\% CI $1.1 \%-4.7 \%, \mathrm{p}=0.03$; Table 2). Patients aged 60 years or older had a better DFS (HR 0.43, 95\% CI 0.20\%-0.94\%, $\mathrm{p}=0.03$; Table 2). Seven patients (18\%) did not experience any local or distant failure after SSRS, at a median follow-up of 75 months (range 20-119 months). On multivariate analysis, higher KPS score (HR $0.37,95 \% \mathrm{CI}$ $0.17 \%-0.81 \%, \mathrm{p}=0.01)$ and older age (HR $0.36,95 \% \mathrm{CI}$ $0.16 \%-0.82 \%, \mathrm{p}=0.02$ ) were also found to be significant (Table 3).

\section{Systemic Therapy Modification-Free Survival}

The median STMFS was 41.2 months (Fig. 3), and the 1- and 2-year rates were $83 \%(95 \%$ CI $67 \%-92 \%)$ and $65 \%(95 \%$ CI $47 \%-79 \%)$, respectively. Patients with a lower KPS score (70-80 vs 90-100) and lumbar or sacral disease (compared with cervical or thoracic) were more likely to experience a modification in systemic therapy (HR 2.9, 95\% CI 1.2\%-6.8\%, $\mathrm{p}=0.02$, and HR 2.4, 95\% CI $1.0 \%-5.3 \%, \mathrm{p}=0.04$, respectively, Table 2). Twentyfour patients $(63 \%)$ had a modification in systemic therapy for progressive disease at a median of 29.0 months (range 3.0-144.4 months). Fourteen patients (37\%) never had an initiation of or modification in systemic therapy (median follow-up 69 months, range 10-145 months). On multivariate analysis, higher KPS score (HR 0.21, 95\% CI $0.08 \%-0.57 \%, \mathrm{p}<0.01$ ), older age (HR $0.21,95 \%$ CI $0.08 \%-0.59 \%, \mathrm{p}<0.01)$, and lumbar/sacral spine location (HR 5.6, 95\% CI $2.1 \%-15 \%, \mathrm{p}<0.01$ ) were found to be significant predictors (Table 3).

\section{Toxicity}

Three patients (8\%) experienced acute toxicity: 2 patients had Grade 2 gastrointestinal (GI) toxicity, and 1 patient had Grade 3 GI toxicity. One patient (3\%) experienced late Grade 4 GI toxicity. This patient received 18 Gy in 1 fraction to T-8, T-8 laminectomy and vertebrectomy, ERBT (30 Gy in 10 fractions) to T7-9, and finally 16 Gy in 1 fraction to T-7, over the course of 2 years, and developed an esophageal fistula and stricture 29 months after the first instance of SSRS (4 months after the last SSRS). One patient (3\%) experienced late Grade 3 myelopathy 12 months after SSRS. Six patients (16\%) developed a compression 


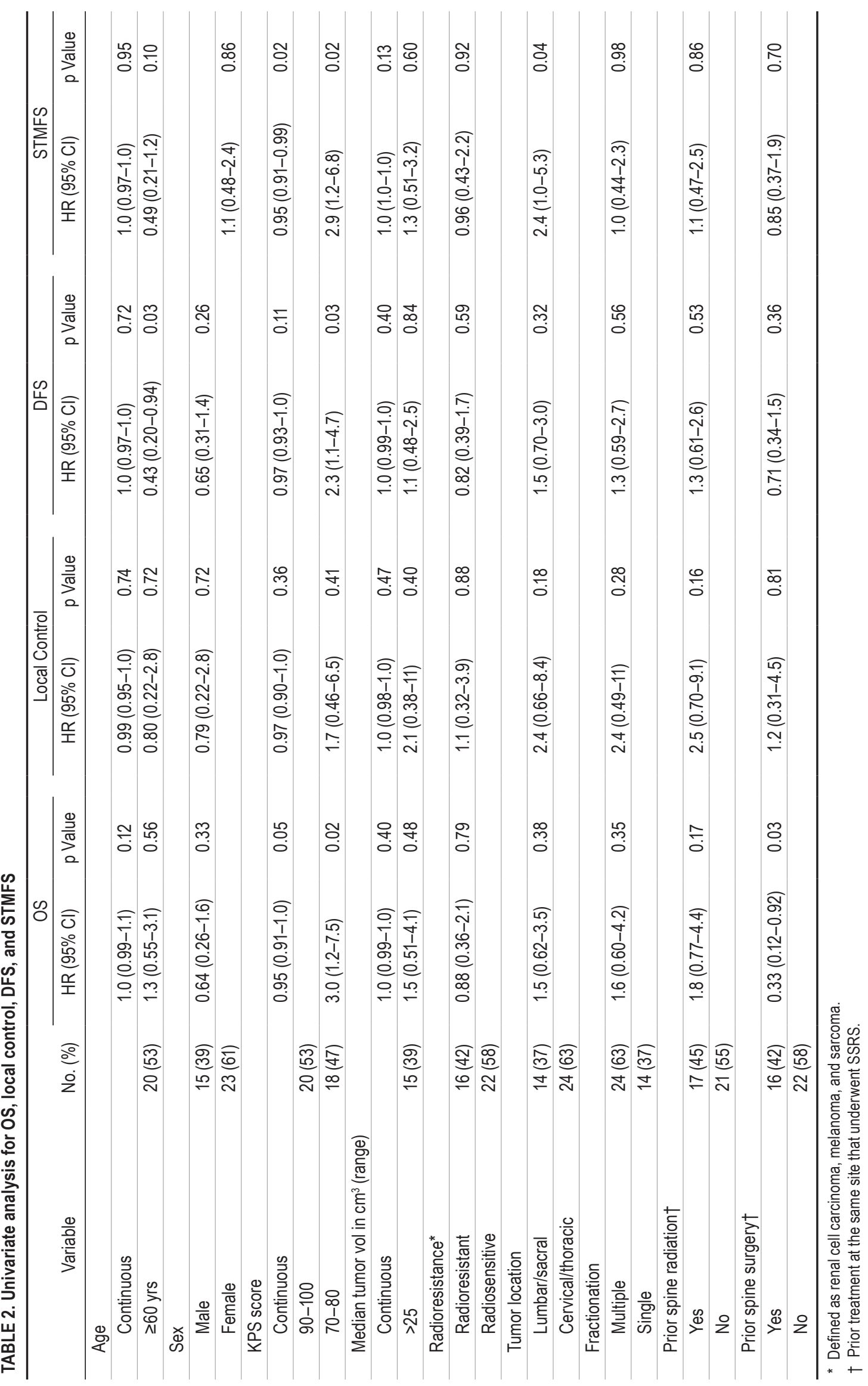



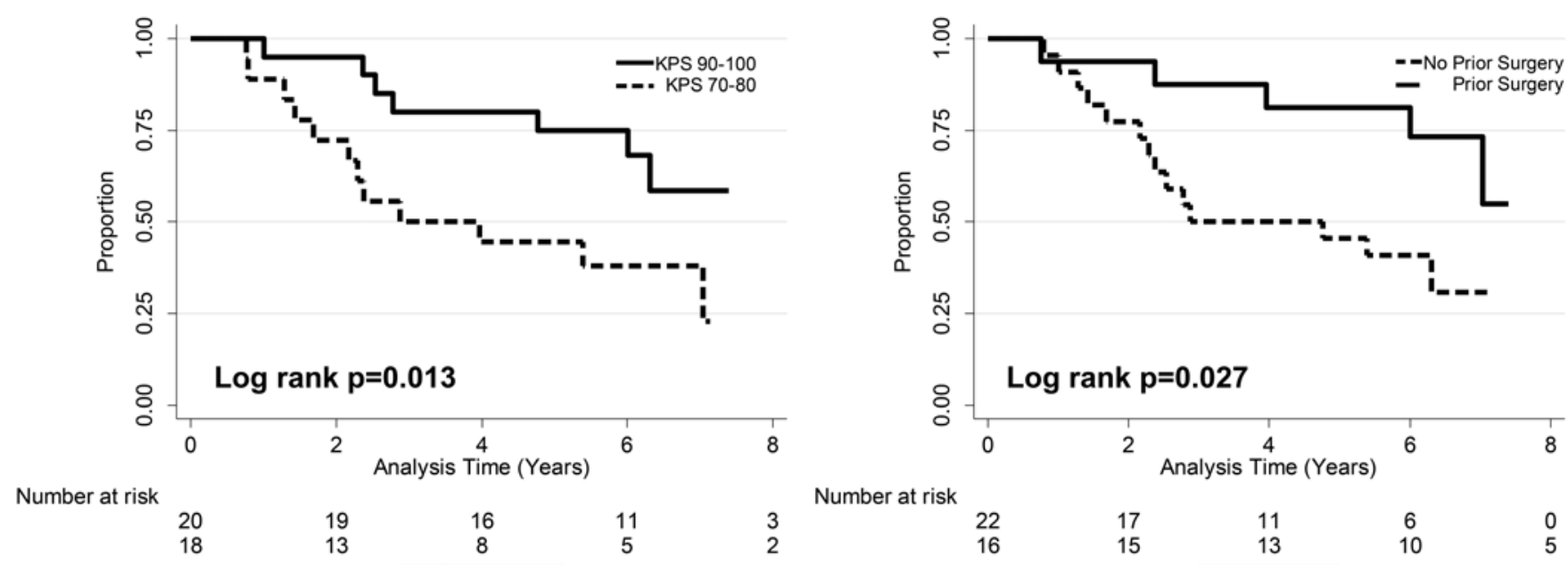

FIG. 2. Kaplan-Meier curves comparing the OS of patients with a KPS score of 90-100 (solid line) versus 70-80 (dashed line; left), and the OS of patients with prior spine surgery (solid line) or no prior spine surgery (dashed line; right).

fracture in the area treated with SSRS at a median of 3.8 months (range 0.7-10.2 months). Five of these patients had symptomatic fractures and subsequently underwent vertebral augmentation procedures. The SSRS doses prescribed in these 6 patients who developed fractures were $24 \mathrm{~Gy}$ in 1 fraction $(\mathrm{n}=3), 18 \mathrm{~Gy}$ in 1 fraction $(\mathrm{n}=1)$, and $27 \mathrm{~Gy}$ in 3 fractions $(\mathrm{n}=2)$.

\section{Discussion}

Our results represent the largest published series of patients $(n=38)$ with oligometastatic spinal metastases treated with SSRS and demonstrate that these patients have an excellent long-term OS with limited toxicity. In addition, we showed that these patients did not need a change in systemic therapy for years following SSRS and that a significant portion had excellent survival after SSRS without the need for systemic therapy.

Significant variability exists in the prognosis for pa- tients treated with SSRS. A recent analysis of patients treated with SSRS identified 7 pretreatment variables that grouped patients into 4 survival groups, with a 5-year overall rate of $66 \%$ for the excellent survival group compared with $0 \%$ for the poor survival group, with 1 of the variables being the SSRS site as the oligometastatic site of disease. ${ }^{28}$ Our results show that as a group, these patients with oligometastatic spine metastases do have an excellent prognosis with a median survival of 76 months and a 5-year survival rate of $60 \%$. Although all of the patients in this series had a KPS score of at least 70, those with a KPS score of 90-100 had a significantly improved survival compared with those with a score of 70-80, consistent with previously reported data. ${ }^{5,28}$ In addition, those patients receiving prior surgery had improved survival in our series, which may represent both the advantages of debulking as well as a healthier selected population.

Patients with oligometastatic disease potentially represent a distinct group with a better prognosis than those

TABLE 3. Multivariate analysis

\begin{tabular}{|c|c|c|c|c|c|c|c|}
\hline \multirow[b]{2}{*}{ Variable } & \multicolumn{2}{|l|}{ OS } & \multirow[b]{2}{*}{ Local Control HR $(95 \% \mathrm{Cl})$} & \multicolumn{2}{|l|}{ DFS } & \multicolumn{2}{|l|}{ STMFS } \\
\hline & $\mathrm{HR}(95 \% \mathrm{Cl})$ & $p$ Value & & $\operatorname{HR}(95 \% \mathrm{Cl})$ & p Value & $\mathrm{HR}(95 \% \mathrm{Cl})$ & $p$ Value \\
\hline \multicolumn{8}{|l|}{ KPS score } \\
\hline $90-100$ & $0.33(0.13-0.84)$ & 0.02 & - & $0.37(0.17-0.81)$ & 0.01 & $0.21(0.08-0.57)$ & $<0.01$ \\
\hline $70-80$ & Reference & & & Reference & & Reference & \\
\hline Prior radiation* & $3.6(1.2-10)$ & 0.02 & NS & - & & - & \\
\hline Prior surgery* & $0.16(0.05-0.52)$ & $<0.01$ & - & - & & - & \\
\hline \multicolumn{8}{|l|}{ Age (yrs) } \\
\hline$\geq 60$ & NS & & - & $0.36(0.16-0.82)$ & 0.02 & $0.21(0.08-0.59)$ & $<0.01$ \\
\hline$<60$ & & & & Reference & & Reference & \\
\hline \multicolumn{8}{|l|}{ Spine location } \\
\hline Lumbar/sacral & - & & NS & - & & $5.6(2.1-15)$ & $<0.01$ \\
\hline Cervical/thoracic & & & & & & Reference & \\
\hline Radiation dose & - & & NS & - & & - & \\
\hline
\end{tabular}

NS = nonsignificant; $-=$ variable not included in the multivariate analysis.

${ }^{*}$ Prior treatment at the same site that underwent SSRS. 
TABLE 4. Patterns of spine failure and salvage therapy

\begin{tabular}{cccccccc}
\hline Type of Spine Failure & No. (\%) & Surgery & Surgery + EBRT & Surgery + SSRS & SSRS & EBRT & Systemic Therapy Alone \\
\hline In-field failure & $10(26)$ & 4 & 2 & - & 1 & - & 3 \\
\hline Out-of-field & $13(34)$ & & & & & & \\
\hline Adjacent level & $3(8)$ & 1 & - & - & 1 & 1 & - \\
\hline Distant level & $10(26)$ & - & - & 1 & 5 & 1 & 3 \\
\hline
\end{tabular}

who develop multiple sites of metastases, and targeted therapy with surgery or ablative radiation may be warranted over the traditional use of only systemic therapy. Several reports of resection of pulmonary, hepatic, or adrenal metastatectomies in select patients have demonstrated excellent long-term survival rates..$^{3,21,29}$ Stereotactic body radiation therapy is a less-invasive alternative to surgery and appears to offer acceptable local control and survival rates for patients with limited metastatic disease. ${ }^{16,17,30} \mathrm{It}$ is currently the subject of several ongoing clinical trials, examining the value of stereotactic body radiation therapy in oligometastatic disease for various primary sites.

However, the literature on the outcomes of SSRS for oligometastatic spinal disease is limited, and, to our knowledge, ours is the largest series to date. Thibault et al. reviewed patients with renal cell cancer treated with SSRS and showed that patients with oligometastatic disease had an improved survival compared with those with more numerous metastases (1-year survival of $84 \%$ vs $53 \%$ ). ${ }^{30}$ Gill et al. reported on 20 patients with oligometastatic spinal metastases treated with Cyberknife SSRS to a median dose of 30 Gy in 5 fractions. ${ }^{14}$ Another report of 4 patients with solitary spine metastases showed excellent survival and control. ${ }^{18}$ Our study stands out as the highest quality evidence, because it is the largest and a reanalysis of Phase I/II trials. In addition, oligometastatic disease has been variably defined in the literature, which makes comparison between studies challenging. Thibault et al. defined it as fewer than 5 sites of metastatic involvement, while Gill et al. did not define it at all. ${ }^{14,30}$ Our analysis specifically was more stringently limited to those patients with

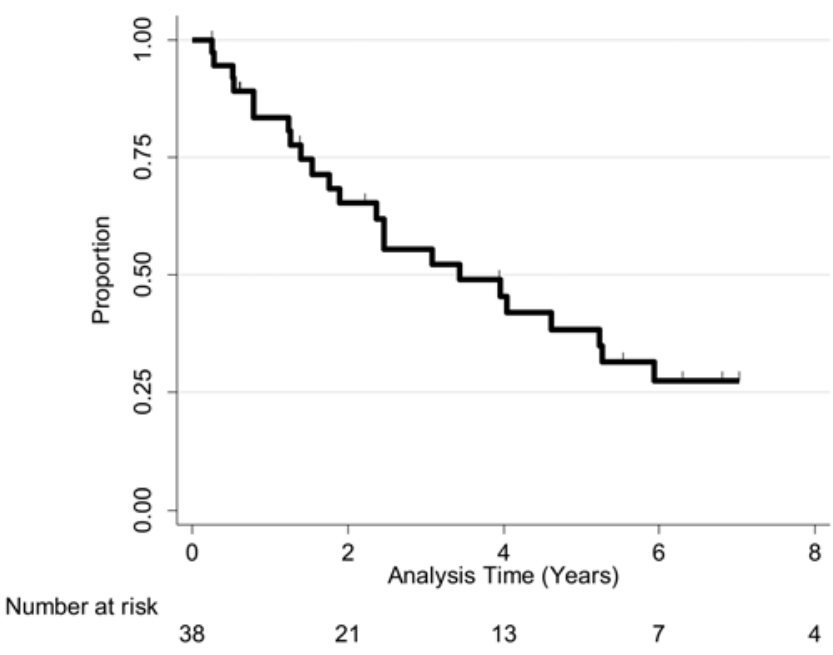

FIG. 3. Kaplan-Meier curve showing the STMFS of all patients. only 1 site of disease: those who either had solitary spine metastases (in $87 \%$ of patients), or those with single spine metastases (13\%) who had prior other metastases that were not progressive. Our series also compared favorably to Gill et al. in terms of survival and local control, with 1- and 2 -year survival rates of $95 \%$ and $84 \%$ compared with $80 \%$ and $57 \%$, and 1- and 2-year local control rates of $85 \%$ and $82 \%$, compared with $80 \%$ and $73 \% .^{14}$ The extended longterm OS of our cohort is likely partially due to the fact that the 2 most common primary sites in our study were renal cell and breast, which have a slower natural course and better prognosis than many other primary sites. Our local control rates were acceptable and comparable to larger series, with a median local failure-free survival of 130.7 months, and a 1- and 2-year rate of $85 \%$ and $82 \%$, respectively. ${ }^{13,31}$ Bishop et al. reported that patients with a GTV biologically equivalent minimum dose (Dmin) of at least 33.4 Gy had significantly better local control, recommending that, when possible, the GTV Dmin should be maintained above $14 \mathrm{~Gy}$ in 1 fraction and 21 Gy in 3 fractions. ${ }^{1}$

In addition to excellent survival and acceptable local control, we have shown that these patients with oligometastatic spine disease had extended time intervals before the initiation of or a modification in systemic therapy, with a median time of 41.2 months. Eighteen percent of patients in this series did not develop any subsequent local recurrences or distant metastases at a follow-up of 75 months and never required systemic therapy (except for 1 patient who started leuprolide shortly after SSRS). This supports the notion that in select patients with oligometastatic spinal metastases that are treated definitively with SSRS, close monitoring without the need for the immediate start of systemic therapy could be a reasonable option. However, it cannot be concluded from this study whether systemic therapy should be initiated sooner or later, because it is possible that it could improve DFS in some patients. In addition, many targeted therapies have low toxicity profiles and may not affect quality of life. A thorough discussion between the medical oncologist and patient should take place regarding this decision.

As an alternative to SSRS, en bloc radical resection is a surgical technique with potentially curative intent that can be offered for oligometastatic spinal disease. ${ }^{7,8,20}$ However, surgical treatment of spine metastases can lead to morbidity and a postoperative complication rate of around $20 \%-30 \% .{ }^{6}$ In particular, older patients have a higher risk of cardiopulmonary and infectious complications from surgery. ${ }^{19,20}$ In contrast, SSRS, as an outpatient noninvasive treatment, is generally very well tolerated and carries minimal morbidity and toxicity, with excellent local control rates. Because most patients will ultimately develop 
further metastases (we reported a median DFS of 19.0 months), SSRS is a practical alternative to en bloc spondilectomy, as it provides durable local control for patients who may ultimately have further progression of disease.

Minimization of late toxicity is especially important after SSRS for these patients with excellent long-term survival. We reported one instance of late Grade 3 myelopathy, and one instance of late Grade 4 GI fistula in a patient who had received multiple courses of radiation and surgery. In addition, $16 \%$ of patients developed compression fractures, consistent with previous reports. ${ }^{2,24}$ There have been variable reports on the dose limitation to the spinal cord using SSRS, with 1 recent analysis reporting a $\leq 5 \%$ risk of myelopathy with a 12.4 Gy Dmax, ${ }^{25}$ or even no reported myelopathy with a Dmax of $14 \mathrm{~Gy} \cdot{ }^{33}$ In general, we limit the spinal cord receiving $10 \mathrm{~Gy}$ to $0.01 \mathrm{~cm}^{3}$ or less for single-fraction SSRS.

Our study is limited by its small sample size, patient heterogeneity, and potential selection bias. However, to our knowledge, it represents the largest analysis of and the highest level of evidence for patients with oligometastatic spine disease treated with SSRS. Because our data are based on a secondary analysis of Phase I/II clinical trials, we have eliminated some of the biases inherent in retrospective analyses, such as information bias and poor follow-up data. Instead, our rigorous and standardized follow-up, with a median follow-up duration of 69 months, allowed us to carefully assess long-term outcomes.

\section{Conclusions}

SSRS for the treatment of oligometastatic spinal metastases is a safe and very effective treatment option with excellent long-term survival, local progression-free survival, and STMFS, and should be offered over palliative radiation regimens for these patients. Further prospective study is warranted to confirm these findings.

\section{References}

1. Bishop AJ, Tao R, Rebueno NC, Christensen EN, Allen PK, Wang XA, et al: Outcomes for spine stereotactic body radiation therapy and an analysis of predictors of local recurrence. Int J Radiat Oncol Biol Phys 92:1016-1026, 2015

2. Boehling NS, Grosshans DR, Allen PK, McAleer MF, Burton AW, Azeem S, et al: Vertebral compression fracture risk after stereotactic body radiotherapy for spinal metastases. J Neurosurg Spine 16:379-386, 2012

3. Casiraghi M, De Pas T, Maisonneuve P, Brambilla D, Ciprandi B, Galetta D, et al: A 10-year single-center experience on 708 lung metastasectomies: the evidence of the "international registry of lung metastases". J Thorac Oncol 6:1373-1378, 2011

4. Chang EL, Shiu AS, Lii MF, Rhines LD, Mendel E, Mahajan A, et al: Phase I clinical evaluation of near-simultaneous computed tomographic image-guided stereotactic body radiotherapy for spinal metastases. Int J Radiat Oncol Biol Phys 59:1288-1294, 2004

5. Chao ST, Koyfman SA, Woody N, Angelov L, Soeder SL, Reddy CA, et al: Recursive partitioning analysis index is predictive for overall survival in patients undergoing spine stereotactic body radiation therapy for spinal metastases. Int J Radiat Oncol Biol Phys 82:1738-1743, 2012

6. Choi D, Crockard A, Bunger C, Harms J, Kawahara N, Mazel $\mathrm{C}$, et al: Review of metastatic spine tumour classification and indications for surgery: the consensus statement of the Global Spine Tumour Study Group. Eur Spine J 19:215-222, 2010

7. Demura S, Kawahara N, Murakami H, Abdel-Wanis ME, Kato S, Yoshioka K, et al: Total en bloc spondylectomy for spinal metastases in thyroid carcinoma. J Neurosurg Spine 14:172-176, 2011

8. Fang T, Dong J, Zhou X, McGuire RA Jr, Li X: Comparison of mini-open anterior corpectomy and posterior total en bloc spondylectomy for solitary metastases of the thoracolumbar spine. J Neurosurg Spine 17:271-279, 2012

9. Foro Arnalot P, Fontanals AV, Galcerán JC, Lynd F, Latiesas XS, de Dios NR, et al: Randomized clinical trial with two palliative radiotherapy regimens in painful bone metastases: $30 \mathrm{~Gy}$ in 10 fractions compared with $8 \mathrm{~Gy}$ in single fraction. Radiother Oncol 89:150-155, 2008

10. Garg AK, Shiu AS, Yang J, Wang XS, Allen P, Brown BW, et al: Phase $1 / 2$ trial of single-session stereotactic body radiotherapy for previously unirradiated spinal metastases. Cancer 118:5069-5077, 2012

11. Garg AK, Wang XS, Shiu AS, Allen P, Yang J, McAleer MF, et al: Prospective evaluation of spinal reirradiation by using stereotactic body radiation therapy: The University of Texas MD Anderson Cancer Center experience. Cancer 117:35093516, 2011

12. Gerszten PC: Spine metastases: from radiotherapy, surgery, to radiosurgery. Neurosurgery 61 (Suppl 1): 16-25, 2014

13. Gerszten PC, Burton SA, Ozhasoglu C, Welch WC: Radiosurgery for spinal metastases: clinical experience in 500 cases from a single institution. Spine (Phila Pa 1976) 32:193-199, 2007

14. Gill B, Oermann E, Ju A, Suy S, Yu X, Rabin J, et al: Fiducial-free CyberKnife stereotactic body radiation therapy (SBRT) for single vertebral body metastases: acceptable local control and normal tissue tolerance with 5 fraction approach. Front Oncol 2:39, 2012

15. Hartsell WF, Scott CB, Bruner DW, Scarantino CW, Ivker RA, Roach M III, et al: Randomized trial of short- versus long-course radiotherapy for palliation of painful bone metastases. J Natl Cancer Inst 97:798-804, 2005

16. Inoue T, Katoh N, Onimaru R, Shirato H: Clinical outcomes of stereotactic body radiotherapy for patients with lung tumors in the state of oligo-recurrence. Pulm Med 2012:369820, 2012

17. Jereczek-Fossa BA, Piperno G, Ronchi S, Catalano G, Fodor C, Cambria R, et al: Linac-based stereotactic body radiotherapy for oligometastatic patients with single abdominal lymph node recurrent cancer. Am J Clin Oncol 37:227-233, 2014

18. Lee S, Chun M, Lee M: Stereotactic body radiotherapy for solitary spine metastasis. Radiat Oncol J 31:260-266, 2013

19. Liang T, Wan Y, Zou X, Peng X, Liu S: Is surgery for spine metastasis reasonable in patients older than 60 years? Clin Orthop Relat Res 471:628-639, 2013

20. Murakami H, Kawahara N, Demura S, Kato S, Yoshioka K, Sasagawa T, et al: Perioperative complications and prognosis for elderly patients with spinal metastases treated by surgical strategy. Orthopedics 33:3, 2010

21. Nordlinger B, Guiguet M, Vaillant JC, Balladur P, Boudjema $\mathrm{K}$, Bachellier P, et al: Surgical resection of colorectal carcinoma metastases to the liver. A prognostic scoring system to improve case selection, based on 1568 patients. Cancer 77:1254-1262, 1996

22. Perrin RG, Laxton AW: Metastatic spine disease: epidemiology, pathophysiology, and evaluation of patients. Neurosurg Clin N Am 15:365-373, 2004

23. Ryu S, Jin R, Jin JY, Chen Q, Rock J, Anderson J, et al: Pain control by image-guided radiosurgery for solitary spinal metastasis. J Pain Symptom Manage 35:292-298, 2008

24. Sahgal A, Atenafu EG, Chao S, Al-Omair A, Boehling N, Balagamwala EH, et al: Vertebral compression fracture after 
spine stereotactic body radiotherapy: a multi-institutional analysis with a focus on radiation dose and the spinal instability neoplastic score. J Clin Oncol 31:3426-3431, 2013

25. Sahgal A, Ma L, Gibbs I, Gerszten PC, Ryu S, Soltys S, et al: Spinal cord tolerance for stereotactic body radiotherapy. Int J Radiat Oncol Biol Phys 77:548-553, 2010

26. Salama JK, Milano MT: Radical irradiation of extracranial oligometastases. J Clin Oncol 32:2902-2912, 2014

27. Singh D, Yi WS, Brasacchio RA, Muhs AG, Smudzin T, Williams JP, et al: Is there a favorable subset of patients with prostate cancer who develop oligometastases? Int J Radiat Oncol Biol Phys 58:3-10, 2004

28. Tang C, Hess K, Bishop AJ, Pan HY, Christensen EN, Yang $\mathrm{JN}$, et al: Creation of a prognostic index for spine metastasis to stratify survival in patients treated with spinal stereotactic radiosurgery: secondary analysis of mature prospective trials Int J Radiat Oncol Biol Phys 93:118-125, 2015

29. Tanvetyanon T, Robinson LA, Schell MJ, Strong VE, Kapoor R, Coit DG, et al: Outcomes of adrenalectomy for isolated synchronous versus metachronous adrenal metastases in non-small-cell lung cancer: a systematic review and pooled analysis. J Clin Oncol 26:1142-1147, 2008

30. Thibault I, Al-Omair A, Masucci GL, Masson-Côté L, Lochray F, Korol R, et al: Spine stereotactic body radiotherapy for renal cell cancer spinal metastases: analysis of outcomes and risk of vertebral compression fracture. J Neurosurg Spine 21:711-718, 2014

31. Wang XS, Rhines LD, Shiu AS, Yang JN, Selek U, Gning I, et al: Stereotactic body radiation therapy for management of spinal metastases in patients without spinal cord compression: a phase 1-2 trial. Lancet Oncol 13:395-402, 2012
32. Witham TF, Khavkin YA, Gallia GL, Wolinsky JP, Gokaslan ZL: Surgery insight: current management of epidural spinal cord compression from metastatic spine disease. Nat Clin Pract Neurol 2:87-94, 116, 2006

33. Yamada Y, Bilsky MH, Lovelock DM, Venkatraman ES, Toner S, Johnson J, et al: High-dose, single-fraction imageguided intensity-modulated radiotherapy for metastatic spinal lesions. Int J Radiat Oncol Biol Phys 71:484-490, 2008

\section{Disclosures}

Dr. Rhines is a consultant for Stryker and Globus.

\section{Author Contributions}

Conception and design: Ghia, Ho, Brown. Acquisition of data: Ghia, Ho, Tang, Deegan, Jonasch, Amini, Wang, Li, Tatsui, Rhines, Brown. Analysis and interpretation of data: Ghia, Ho, Tang, Allen, Wang, Brown. Drafting the article: Ghia, Ho, Allen. Critically revising the article: all authors. Reviewed submitted version of manuscript: all authors. Approved the final version of the manuscript on behalf of all authors: Ghia. Statistical analysis: Allen. Administrative/technical/material support: all authors. Study supervision: Ghia.

\section{Correspondence}

Amol J. Ghia, Department of Radiation Oncology, The University of Texas MD Anderson Cancer Center, 1515 Holcombe Blvd., Unit 0097, Houston, TX 77030. email: ajghia@mdanderson.org. 